\title{
ArcheoSciences
}

Revue d'archéométrie

\section{Distribution spatiale des objets archéologiques et mouvements de matière à l'échelle des versants cultivés : le cas de Mougon (Indre-et-Loire, France)}

Spatial Distribution of Archaeological Artefacts and Soils Redistribution at the Scale of Cultivated Slopes in Mougon (Indre-et-Loire, France)

Anne Moreau, Sylvie Morice, Sébastien Salvador-Blanes et Hocine Bourennane

\section{OpenEdition \\ Journals}

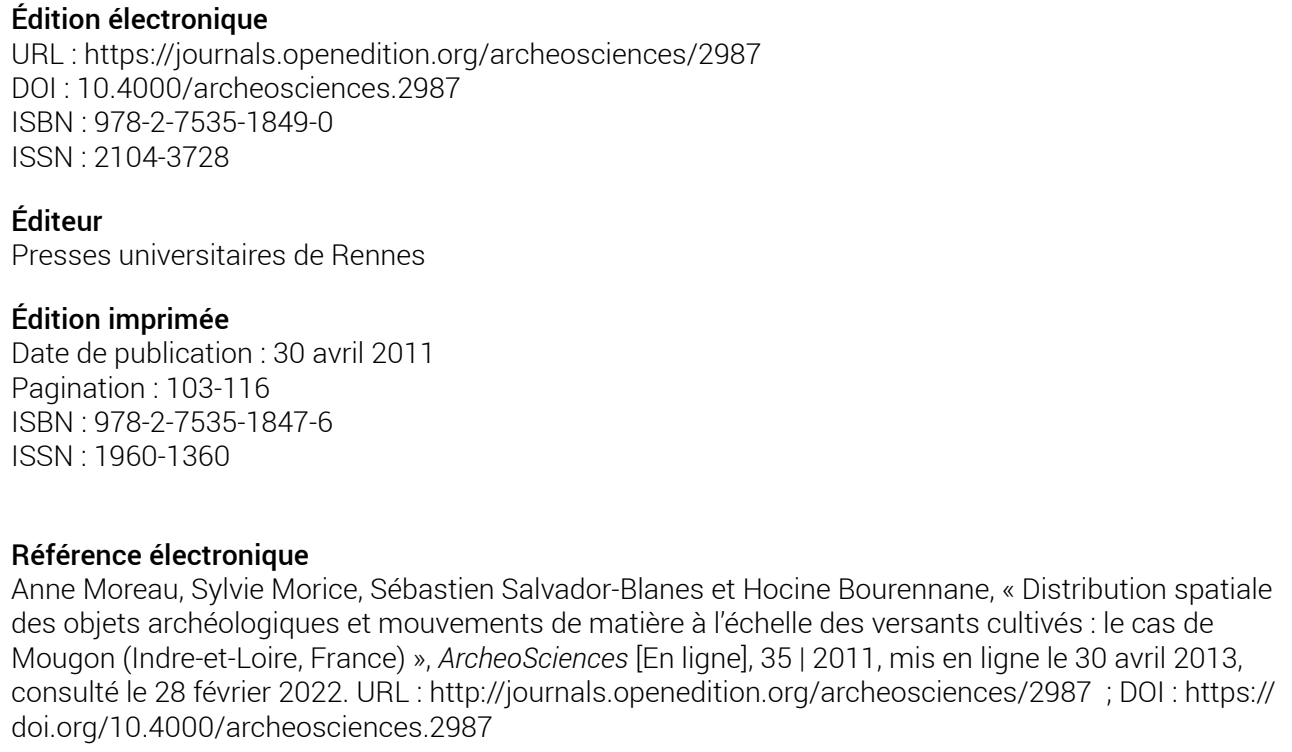
des objets archéologiques et mouvements de matière à l'échelle des versants cultivés : le cas de Mougon (Indre-et-Loire, France) », ArcheoSciences [En ligne], 35 | 2011, mis en ligne le 30 avril 2013, consulté le 28 février 2022. URL : http://journals.openedition.org/archeosciences/2987 ; DOI : https:// doi.org/10.4000/archeosciences.2987 


\title{
Distribution spatiale des objets archéologiques et mouvements de matière à l'échelle des versants cultivés : le cas de Mougon (Indre-et-Loire, France)
}

\author{
Spatial Distribution of Archaeological Artefacts and Soils Redistribution \\ at the Scale of Cultivated Slopes in Mougon (Indre-et-Loire, France)
}

\author{
Anne Moreau*, Sylvie Morice**, Sébastien Salvador-Blanes*** \\ et Hocine BourennanE****
}

\begin{abstract}
Résumé : Cette contribution pose la question de l'utilisation de la géostatistique pour l'interprétation de la répartition des artefacts recueillis en prospection archéologique. L'expérience a été réalisée à partir des données issues des prospections réalisées à Mougon (commune de Crouzilles, Indre-et-Loire, France) dans le cadre d'un projet retenu par l'Action Concertée Incitative "Terrain, Technique, Théorie » du ministère de la recherche. L'objectif initial visait à établir une distinction entre les concentrations d'artefacts susceptibles de signaler la présence de structures archéologiques sous-jacentes et les zones de répartition spatiale aléatoire des artefacts, liée au fumage des terres cultivées et aux processus postdépositionnels. Après le tri, le comptage et l'enregistrement informatique du mobilier par catégories chronologiques et fonctionnelles, les artefacts collectés en surface ont fait l'objet d'un traitement géostatistique destiné à faciliter la lecture et l'interprétation de leur répartition : la distribution des artefacts a été étudiée au moyen des méthodes géostatistiques de krigeage ordinaire et de la simulation conditionnelle. L’exploitation des données de surface par le biais de la géostatistique a permis, d'une part, de circonscrire précisément l'emplacement des structures archéologiques potentielles et d'autre part, de révéler l'importance des processus post-dépositionnels dans la redistribution des artefacts en surface. Cette expérience est également à l'origine d'une réflexion sur la pertinence des unités de collecte et de représentation du mobilier de prospection.
\end{abstract}

Abstract: This contribution aims at discussing the use of geostatistics for the interpretation of the spatial surface distribution of archaeological artefacts. The experience was carried out with the artefacts collected in Mougon (Crouzilles, Indre-et-Loire, France) for the ACI TTT project of the French Ministry of Research. The initial purpose of the study was to distinguish the concentrations of artefacts indicating the presence of buried archaeological remains from a random spatial distribution of artefacts due to manuring and post-depositional processes. After all the artefacts were sorted out, counted and recorded, a geostatistical analysis has been performed to interpret the spatial distribution of the surface artefacts: ordinary kriging and conditional simulation were used. The use of geostatistics allowed a precise delimitation of archaeological features. It also revealed the influence of post-depositional processes on the spatial distribution of artefacts. Furthermore, this experiment led us to re-estimate the relevance of the units we used to collect and represent the survey data.

Mots clé : Artefacts, distribution spatiale, géostatistique, krigeage, prospection, structure archéologique

Keywords: archaeological faeture, artifacts, geostatistic, kriging, spatial distribution, survey

* Docteur, Inrap, Laboratoire Archéologie et Territoires UMR 7324 CITERES, MSH VILLES ET TERRITOIRES, 35 Allée Ferdinand de Lesseps, 37204 TOURS cedex 03, Tours, France. (anne.morean@inrap.fr)

** Doctorante, EA Géo-hydrosystèmes Continentaux, Université François-Rabelais, Faculté des Sciences et des Techniques - Parc Grandmont, avenue Monge, 37200 Tours, France. (morice.sylvie@gmail.com)

*** Docteur, EA Géo-hydrosystèmes Continentaux, Université François-Rabelais, Faculté des Sciences et des Techniques - Parc Grandmont, 37200 Tours, France. (salvador@univ-tours.fr)

**** Docteur, INRA - 2163 avenue de la Pomme-de-Pin, CS 40001, Ardon, 45075 Orléans cedex, France. (hocine.bourennane@orleans.inra.fr) 


\section{INTRODUCTION}

La démarche qui sous-tend cette étude est méthodologique : nous exposons ici les méthodes et les résultats de l'analyse géostatistique effectuée à partir de données de prospection pédestre dans le but de débattre de l'intérêt d'une telle étude pour l'interprétation de la distribution des artefacts au sol.

Les artefacts présents à la surface des terres cultivées témoignent d'activités anthropiques diverses - occupations in situ plus ou moins pérennes, exploitation du milieu - que les archéologues tentent d'appréhender à travers les données collectées lors des prospections systématiques (Foard, 1978; Ferdière et Zadora-Rio, 1986; Schofield, 1991). Dans le cadre d'un projet piloté par le Laboratoire Archéologie et Territoires de Tours et développé au sein de l'Action Concertée Incitative "Terrain, Technique et Théorie » du ministère de la Recherche, une campagne de prospection en carroyage associant un ramassage à vue, des prospections géophysiques, géochimiques et un relevé topographique a été effectuée à proximité du bourg de Mougon dans la commune de Crouzilles (Indre-et-Loire, fig. 1).

À l'issue de la prospection de surface, plus de 73000 artefacts ont été collectés sur une douzaine d'hectares (ZadoraRio et al., 2008). L'abondance du mobilier ramassé et la précision de l'unité de collecte et d'enregistrement des données (cellules de $3 \mathrm{~m}$ x $10 \mathrm{~m}$, $c f$. infra) ont permis d'exploiter le mobilier de surface selon deux directions complémentaires dictées par des problématiques archéologiques et pédologiques : il s'agissait, d'une part, de distinguer les concentrations d'artefacts susceptibles de signaler la présence de vestiges archéologiques sous-jacents des zones de répartition aléatoire de mobilier liées à l'amendement des champs cultivés et aux phénomènes post-dépositionnels et d'autre part,

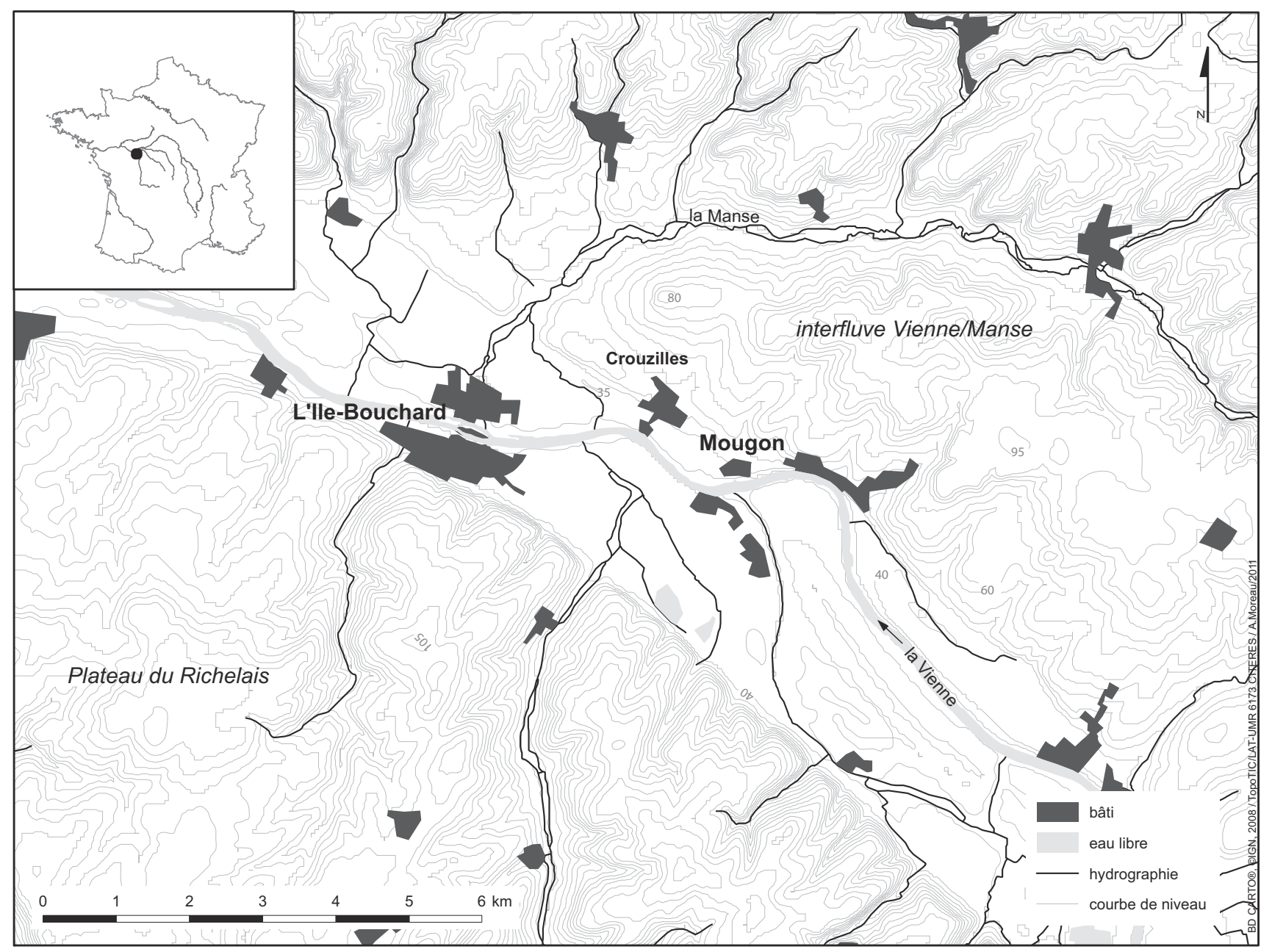

Figure 1: La vallée de la Vienne à Mougon (Indre-et-Loire)

Figure 1: The Vienne valley in Mougon (Indre-et-Loire) 
d'utiliser le mobilier de surface pour caractériser la redistribution de la matière à l'échelle de versants cultivés.

Dans cette optique, une étude géostatistique de la répartition du mobilier de surface a été réalisée dans le cadre du programme ECLIPSE engagé au sein des laboratoires de I'UMR 6113 ISTO à l'Université de Tours et du Laboratoire Archéologie et Territoires de Tours.

\section{Collecte et traitement DES DONNÉES ARCHÉOLOGIQUES}

\section{La prospection}

\section{Localisation et description de la zone de prospection intensive}

La zone de prospection a été implantée au nord du hameau de Mougon qui borde la Vienne en rive droite. Elle englobe trois parcelles cadastrales distinctes : la Butte des Moulins au nord, la Robinerie au centre et une parcelle dite "le clos" (ancien clos de vigne) au sud. Ces trois parcelles ne sont pas jointives : la butte des Moulins est séparée de la Robinerie par la route départementale 760 au nord et la Robinerie est séparée de la parcelle du clos par l'ancienne voie de chemin de fer devenue un simple chemin de desserte locale après l'abandon de la ligne dans la première moitié du $\mathrm{xx}^{\mathrm{e}}$ siècle (fig. 2).

La zone étudiée est caractérisée par un substrat composé d'affleurements de roches carbonatées dans la partie nord et de formations fluviatiles quaternaires dans le clos en bas du versant en rive droite de la vallée de la Vienne (Alcaydé, 1977). On trouve ainsi à la base de la séquence stratigraphique la craie blanche à Inocérames, attribuée au Turonien inférieur (C3a), sur laquelle repose la craie micacée (tuffeau blanc), attribuée au Turonien moyen (C3b). Ces formations affleurent sur la plus grande partie de la zone étudiée (fig. 3). L'extrémité sud de la zone prospectée est située sur des alluvions fluviatiles anciennes de la Vienne (Fw, Fx), correspondant respectivement à deux terrasses fluviatiles constituées de sables et de galets (silex et roches cristallines).

La morphologie de la zone prospectée est représentative de la topographie en " marches d'escalier " des terrasses fluviatiles qui caractérise l'interfluve Vienne-Manse : la parcelle du clos est située sur une basse terrasse de la Vienne en limite du lit majeur de la rivière à environ $44 \mathrm{~m}$ d'altitude (fig. 3). La Robinerie et la Butte des Moulins, point culminant de l'aire étudiée, constituent un versant de pente moyenne (environ $7 \%)$ correspondant aux coteaux carbonatés de la Vienne dont l'altitude varie entre 45 et 66 mètres NGF. Notons que l'extrémité ouest de la zone prospectée est occupée par un talweg orienté nord-est/sud-ouest (fig. 4). L'intensité de la pente est très variable : elle est comprise entre 0,1 et $31,4 \%$. Les valeurs de pente les plus élevées sont localisées sur la Butte des Moulins (fig. 4).

\section{Implantation de la zone de prospection}

La zone carroyée couvre une superficie approximative de douze hectares. Le choix d'implantation de la zone de prospection a été dicté d'une part, par le contexte archéologique et d'autre part, par les indices archéologiques détectés sur les photographies aériennes.

Le site de Mougon a été signalé pour la première fois en 1848 pour sa nécropole du haut Moyen Age (Bourassé, 1854) puis en 1871 pour ses fours de potiers antiques (Chevalier, 1871). Depuis, le site et ses abords immédiats ont fait l'objet de multiples opérations archéologiques qui ont permis de mieux cerner l'occupation du secteur : au total, plus d'une quarantaine de fours de potiers ainsi que des structures diverses organisées selon un réseau de voies ont été détectés. L'abondance des structures artisanales associées à des structures domestiques a permis d'identifier le site à une agglomération secondaire antique. On sait, d'après les fouilles et les prospections de surface, que la production de l'officine débute dans le courant de la première moitié du $\mathrm{I}^{\text {er }}$ siècle après J.-C. et ne perdure pas au-delà de la fin du $\mathrm{II}^{\mathrm{e}}$ siècle- début du $\mathrm{III}^{\mathrm{e}}$ siècle après J.-C. (Ferdière, 1999; Moreau, 2008). L'extension de la nécropole du haut Moyen Age a été reconnue par des fouilles et des prospections géophysiques; elle s'étend sur près de trois hectares autour l'église Saint Pierre de Mougon édifiée au v siècle selon Grégoire de Tours (Moreau, 2008).

Outre le contexte archéologique favorable à une recherche de terrain, plusieurs indices issus de la photo-interprétation ont dicté le choix de l'emplacement de la zone de prospection : des anomalies circulaires visibles sur la Butte des Moulins avaient été interprétées comme une enceinte protohistorique potentielle (Couderc, 1982; Dubois, 1985). Par ailleurs, une " tache claire " détectée dans la parcelle de la Robinerie suggérait la présence d'un bâtiment gallo-romain (Dubois, 1985).

\section{Le ramassage de surface}

Deux carroyages composés de carrés de $30 \mathrm{~m}$ x $30 \mathrm{~m}$ ont été implantés sur les trois parcelles sélectionnées. Au total, 133 carrés ont été prospectés. Ceux-ci ont servi de cadre à tous les modes de prospection (fig. 2).

Pour le ramassage de surface, chaque carré de $30 \mathrm{~m}$ x $30 \mathrm{~m}$ a été subdivisé en dix traverses et trois transects, formant ce que nous avons appelé des " cellules " de trois mètres sur dix mètres (fig. 1). La taille et la forme de la cellule avaient été 


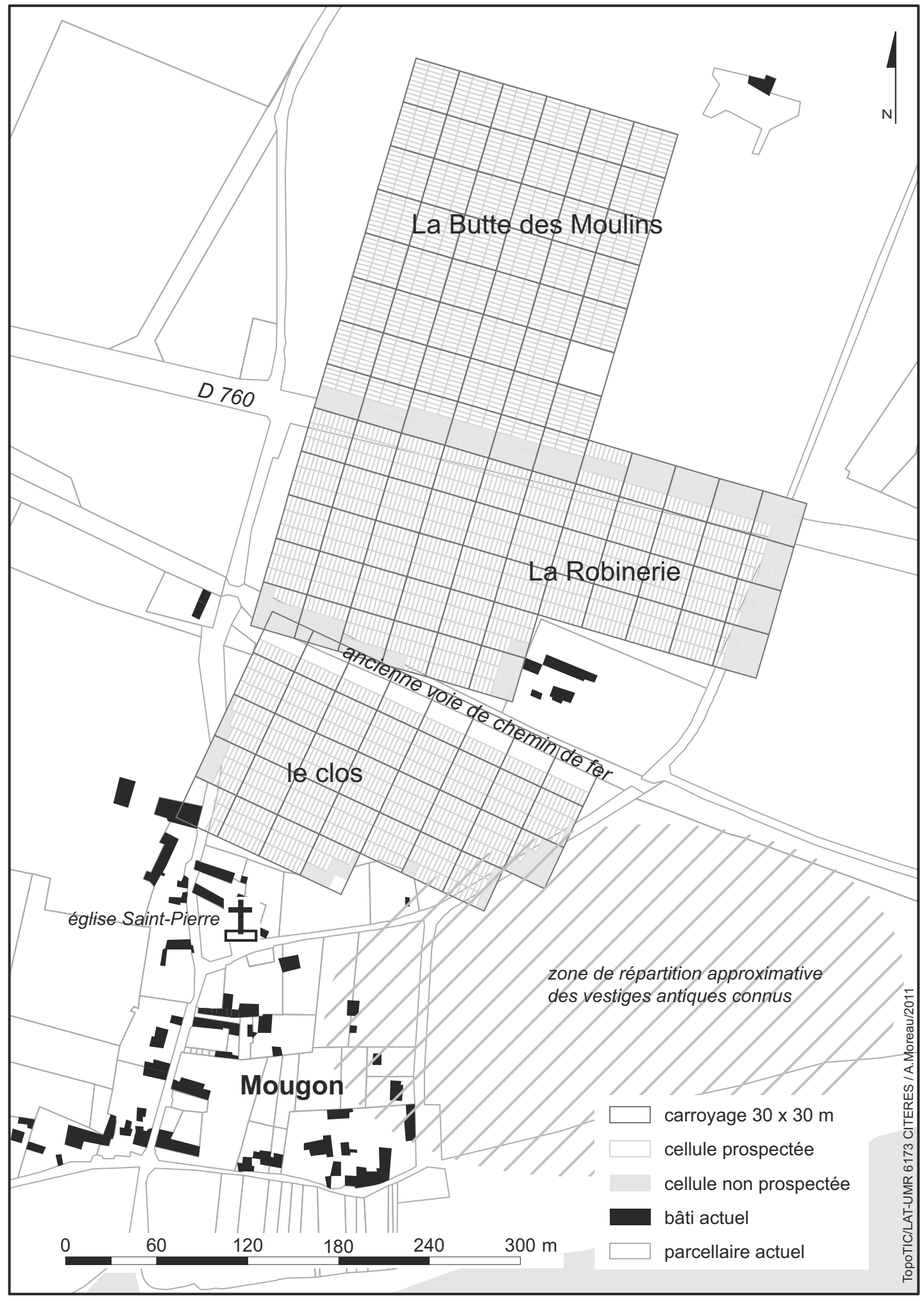

Figure 2 : La zone de prospection.

Figure 2: The surveyed area. 


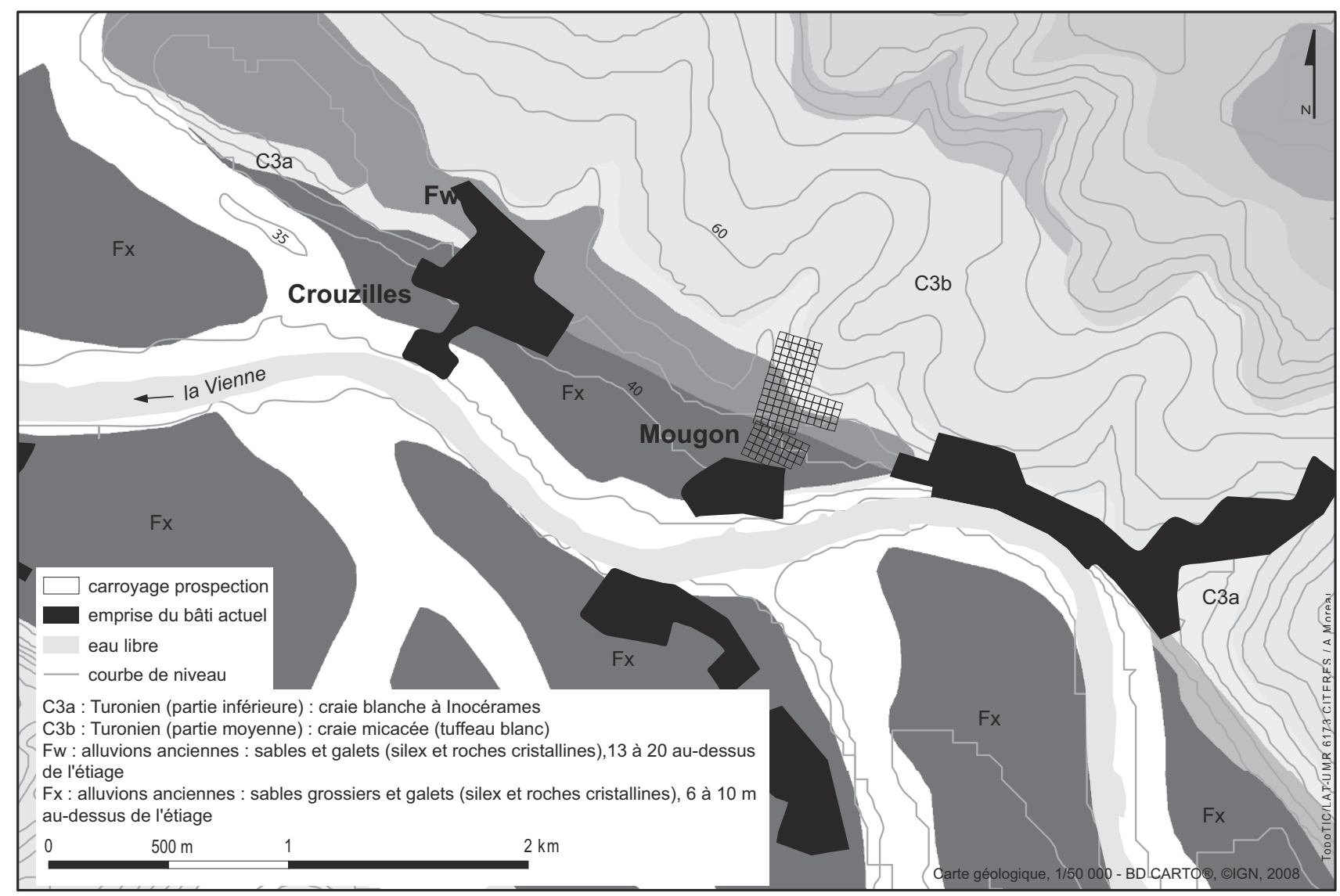

Figure 3 : Extrait de la carte géologique 1/50 000.

Figure 3: Extract from the 1/50000 geological map.

définies dans le cadre d'une prospection précédente réalisée sur l'un des terrains d'étude du projet localisé en Angleterre. Afin de rendre les résultats des prospections comparables d'une étude de cas à l'autre, il a été décidé d'appliquer partout les mêmes procédés de prospection (mailles de collecte et méthodes de prospection identiques). La cellule de $3 \mathrm{~m} \mathrm{x}$ $10 \mathrm{~m}$ représente donc l'unité de ramassage, d'enregistrement et de représentation des données collectées à Mougon.

Dans chaque cellule, la totalité du mobilier a été ramassé : les Terres Cuites Architecturales, l'ardoise, les roches exogènes, la céramique, les ossements, les silex taillés, le verre et les objets divers ont été systématiquement collectés sans sélection intentionnelle lors du ramassage. Tous les artefacts ont ensuite été triés par catégorie, comptés en Nombre de Restes (NR) et pesés.

15 catégories ont été définies : sept catégories de matériaux de construction - tegulae, imbrices, tuile plates ou tuiles à crochet, tuiles mécaniques, terres cuites architecturales (TCA) indéterminées, briques/carreaux et ardoises - et huit catégories chronologiques (en siècles) de céramique - [céramique protohistorique], $\left[\mathrm{I}^{\mathrm{er}}-\mathrm{III} \mathrm{e}^{\mathrm{e}}\right],\left[\mathrm{IV}^{\mathrm{e}}-\mathrm{VII}{ }^{\mathrm{e}}\right],\left[\mathrm{VIII}^{\mathrm{e}}-\mathrm{XI}^{\mathrm{e}}\right],\left[\mathrm{XII}^{\mathrm{e}}-\right.$ $\left.\mathrm{XIII}{ }^{\mathrm{e}}\right],\left[\mathrm{XIV}^{\mathrm{e}}-\mathrm{XV}^{\mathrm{e}}\right],\left[\mathrm{XVI}^{\mathrm{e}}-\mathrm{XVII}^{\mathrm{e}}\right]$ et $\left[\mathrm{XVII}^{\mathrm{e}}\right.$ et plus].

\section{Traitement géostatistiQue DeS DONNÉES}

\section{Statistiques}

Sur les 73000 et quelques restes collectés, on a recensé 37372 fragments de matériaux de construction et 34415 tessons de céramique, toutes périodes confondues. Cela représente en moyenne plus de 4000 tessons par hectare. Le nombre d'artefacts est très variable d'une catégorie de mobilier à l'autre : 5 catégories sur 15 concentrent $90 \%$ de la totalité des artefacts collectés. Ainsi, la céramique antique des $\mathrm{I}^{\text {er-}}-\mathrm{III}^{\mathrm{e}}$ siècles représente plus de $40 \%$ de la totalité des artefacts et $90 \%$ de l'ensemble des tessons de céramique. 


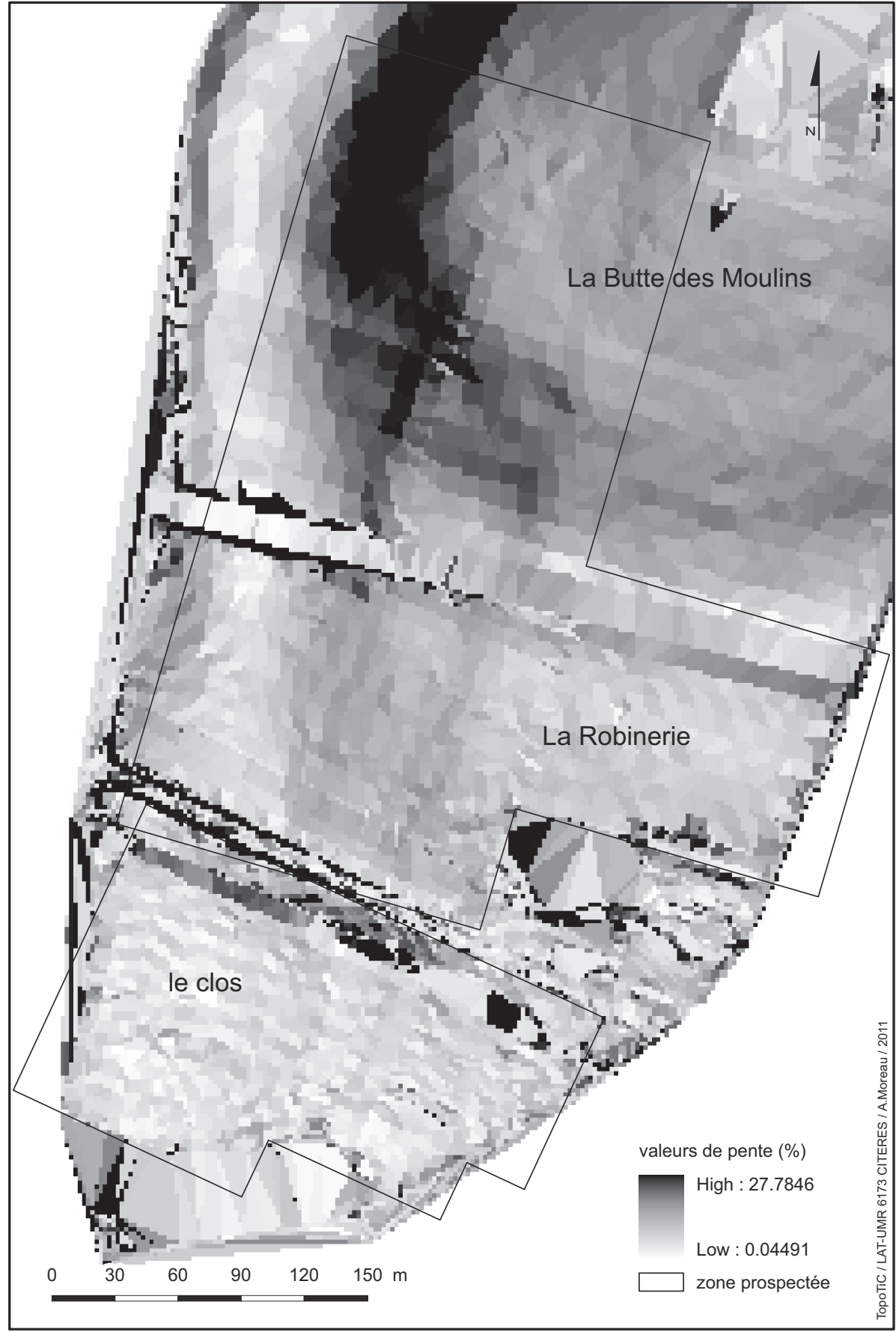

Figure 4 : Intensité de la pente, zone de prospection.

Figure 4: Slope intensity, surveyed area.

\section{Présentation des méthodes}

Deux méthodes ont été employées pour décrire la distribution spatiale des artefacts : la simulation conditionnelle a été utilisée pour délimiter les zones de dépassement de seuils d'artefacts tandis que l'estimation par krigeage a été mise en œuvre pour cartographier la répartition des artefacts et les teneurs géochimiques en vue de leur confrontation avec le zonage établi par simulation conditionnelle.

\section{Estimation spatiale}

L'estimation spatiale comporte deux étapes : l'analyse variographique, qui permet de déterminer si les variables sont spatialement structurées, c'est-à-dire si la valeur prise 
par une variable est corrélée avec les valeurs voisines et le ou les krigeages, outils d'estimation de la variable considérée en un point où aucune valeur n'est disponible.

\section{L'ANALYSE VARIOGRAPHIQUE ET L'AJUSTEMENT DU MODÈLE AU VARIOGRAMME}

Elle consiste à calculer un variogramme expérimental destiné à être ensuite ajusté par une structure théorique.

Pour mesurer la variabilité à différentes échelles d'une variable régionalisée $\mathrm{z}(\mathrm{x})$, on réalise une mesure de dissemblance entre deux données $\mathrm{z}_{1}$ et $\mathrm{z}_{2}$ situées en deux points $\mathrm{x}_{1}$ et $\mathrm{x}_{2}$ d'un domaine spatial (D). Cette dissemblance entre deux valeurs, désignée par $\gamma^{*}$, est définie par le demi écart quadratique.

$$
\gamma^{*}=\frac{\left(z_{2}-z_{1}\right)^{2}}{2}
$$

On fait dépendre la dissemblance $\gamma^{*}$ de la distance et de l'orientation d'une paire de points, décrits par le vecteur $\mathrm{x}_{2}-\mathrm{x}_{1}$, indifféremment de la position de la paire dans le domaine étudié.

En formant la moyenne des dissemblances $\gamma^{*}$ entre les valeurs pour les $n_{\mathrm{h}}$ paires de points reliées par un vecteur h donné pour une maille donnée (avec, le cas échéant, une certaine tolérance sur la longueur et l'angle du vecteur), on obtient la notion de variogramme expérimental.

$$
\gamma^{*}(\mathbf{h})=\frac{1}{2 n_{\mathbf{h}}} \sum_{\alpha=1}^{n_{\mathrm{h}}}\left[z\left(\mathbf{x}_{\alpha}+\mathbf{h}\right)-z\left(\mathbf{x}_{\alpha}\right)\right]^{2} .
$$

Habituellement, on constate que la dissemblance des valeurs augmente en moyenne avec l'éloignement spatial des points de mesure et atteint fréquemment un palier aux grandes distances. Lorsque la dissemblance moyenne des valeurs est constante pour toutes les distances, on constate l'absence totale de structuration spatiale des valeurs. A l'inverse, une pente non nulle du variogramme près de l'origine indique une structuration des données. Un changement soudain de la pente du variogramme indique une structuration complexe des valeurs à plusieurs échelles spatiales.

Trois indicateurs permettent de caractériser un variogramme expérimental :

- l'effet de pépite (nugget effect) : il s'agit de la valeur de la variance pour une distance $h$ tendant vers zéro; en théorie, la variance est nulle pour $h=0$ mais pour les plus petites valeurs de $\mathrm{h}$ ce n'est pas le cas en pratique et $\gamma$ peut être significativement différent de zéro. L'écart est appelé l'« effet de pépite »; il est interprété comme le résultat d'erreurs de mesure ou d'une variabilité spatiale présente à une distance inférieure au pas d'échantillonnage (sous échantillonnage) plus il est faible, mieux la structure spatiale des données est décrite avec le maillage adopté;

- le palier (sill) est la valeur de la variance à partir de laquelle le variogramme ne croît plus;

- la portée (range) correspond à la distance à partir de laquelle un pallier est atteint.

La recherche d'anisotropie éventuelle de la variable étudiée (comportement différent de la variable étudiée suivant l'orientation) est diagnostiquée à travers l'estimation de variogrammes directionnels.

Un modèle théorique est ensuite ajusté au variogramme expérimental : il s'agit d'une fonction dont l'expression mathématique simple qui s'adapte au mieux aux points du variogramme expérimental. Cet ajustement est nécessaire en vue de l'estimation spatiale.

\section{ESTIMATION PAR KRIGEAGE}

Lestimation par krigeage est la deuxième étape d'une analyse géostatistique classique. Dans le cadre de cette étude, le krigeage ordinaire est utilisé pour les estimations de la répartition spatiale des différentes variables.

Le krigeage ordinaire correspond à l'estimation d'une valeur inconnue en un point. L'opération est répétée à chaque noeud d'une grille régulière recouvrant le domaine étudié.

Soit $\mathrm{x}_{\alpha}$, le nombre de points échantillonnés dans un domaine $\mathrm{D}$. On désire estimer une valeur $\mathrm{z}^{*}$ en un point $\mathrm{x}_{0}$. La nouvelle valeur $\mathrm{z}^{*}$ est estimée par une moyenne pondérée de données $\mathrm{z}\left(\mathrm{x}_{\alpha}\right)$

$$
z^{*}\left(\mathbf{x}_{0}\right)=\sum_{\alpha=1}^{n} w_{\alpha} z\left(\mathbf{x}_{\alpha}\right) .
$$

Les $\mathrm{n}$ pondérateurs $\mathrm{w}_{\mathrm{a}}$ sont calculés de façon à assurer que l'estimation $\mathrm{z}^{*}\left(\mathrm{x}_{0}\right)$ soit sans biais :

$$
\left(\sum_{a=1}^{n} w_{a}=1\right)
$$

et que la variance de l'erreur d'estimation soit minimale. Le calcul des pondérateurs ne dépend pas de la valeur de la variable étudiée mais uniquement de la structure spatiale de la variable et de la distance géographique des points observés aux points à estimer. C'est donc 
l'ajustement du variogramme préalablement estimé qui permet d'affecter les pondérateurs aux points observés. Ainsi, si le variogramme est de type pépitique, indiquant une distribution aléatoire, le poids affecté à l'ensemble des points observés est le même. On ne privilégie pas les points les plus proches du point à estimer. Si la variable étudiée présente une structure spatiale, le point le plus proche du point à estimer se voit affecter le pondérateur le plus important.

Une fois estimée la valeur de la propriété en tout point de l'espace, il est possible de créer une carte représentant la répartition de la propriété dans l'espace. En général, l'estimation est réalisée aux nœuds d'une grille de maille carrée qui recouvre le domaine étudié, pour les variables qui présentent un variogramme structuré. La taille de la maille a été fixée à deux mètres de côté.

\section{VALIDATION CROISÉE}

La validation croisée est un outil simple pour vérifier différentes hypothèses telles que :

(1) le type de variogramme théorique ajusté au variogramme expérimental et ses paramètres;

(2) le voisinage choisi pour le krigeage.

Dans la procédure de validation croisée, une valeur $\mathrm{z}\left(\mathrm{x}_{\alpha}\right)$ est retirée du jeu de données et sa valeur $\mathrm{z}^{*}\left(\mathrm{x}_{[\alpha]}\right)$ est estimée au point $\mathrm{x}_{\alpha}$ par krigeage ordinaire à partir des $(\mathrm{n}-1)$ autres valeurs. La notation $[\alpha]$ signifie que la donnée existant en $\mathrm{x}_{\alpha}$ n'a pas été utilisée pour estimer $\mathrm{z}^{*}\left(\mathrm{x}_{[\alpha]}\right)$.

La différence entre la valeur mesurée et la valeur estimée $\mathrm{z}\left(\mathrm{x}_{\alpha}\right)-\mathrm{z}^{*}\left(\mathrm{x}_{[\alpha]}\right)$ nous informe sur la capacité du krigeage à estimer une donnée à partir des autres données dans le voisinage. Si les erreurs de validation croisée moyennes sont proches de zéro,

$$
\begin{gathered}
\frac{1}{n} \sum_{\alpha=1}^{n}\left[z\left(\mathbf{x}_{\alpha}\right)-z\left(\mathbf{x}_{[\alpha]}\right)\right] \models 0, \\
\left(\frac{z\left(\mathbf{x}_{\alpha}\right)-z^{*}\left(\mathbf{x}_{[\alpha]}\right)}{\sigma_{k}}\right)
\end{gathered}
$$

il n'y a pas de biais systématique. Au contraire, une erreur moyenne négative (ou positive) représente une sur- (sous-) estimation systématique. L'écart-type du krigeage $\sigma_{k}$ représente l'erreur estimée par le modèle quand le krigeage est réalisé en $\mathrm{x}_{\alpha}$ (la valeur en $\mathrm{x}_{\alpha}$ est supposée inconnue).

En divisant l'erreur de validation croisée par $\sigma_{\mathrm{k}}$ : on peut comparer l'erreur effective avec l'erreur théorique. Si la moyenne des carrés des erreurs standardisées de validation croisée est proche de l'unité

$$
\frac{1}{n} \sum_{\alpha=1}^{n} \frac{\left[z\left(\mathbf{x}_{\alpha}\right)-z\left(\mathbf{x}_{[\alpha}\right)^{2}\right]}{\sigma_{k}^{2}} \cong 1 \text {, }
$$

l'erreur d'estimation effective est égale en moyenne à l'erreur prévue par le modèle. Cette quantité donne une idée de l'adéquation du modèle et de ses paramètres.

\section{Simulation conditionnelle}

La détermination des structures archéologiques potentielles a été effectuée par une simulation conditionnelle gaussienne (SCG, Castrignano et Buttafuoco, 2004; Cinnirella et al., 2005). Cette méthode permet de reproduire le spectre de variation du phénomène étudié : elle ne minimise pas la variance locale, et contrairement à l'estimation par krigeage ordinaire qui ne donne qu'une réalisation au nœud de la grille, plusieurs réalisations apparaissent au niveau de chaque nœud.

La simulation conditionnelle procède de la façon suivante : on considère $\mathrm{N}$ valeurs conditionnantes gaussiennes choisies sur la base de la portée du variogramme et $\mathrm{N}$ points à simuler. On produit d'abord un chemin aléatoire permettant de "visiter " à tour de rôle chacun des n points à simuler. Pour chaque point, on effectue le krigeage simple de ce point en utilisant l'ensemble des $\mathrm{N}$ points conditionnants et tous les points ayant déjà été simulés. Puisqu'on a choisi l'hypothèse d'une répartition gaussienne, la valeur krigée et la variance de krigeage correspondent respectivement à l'espérance conditionnelle et la variance conditionnelle de la variable aléatoire au point visité. On ajoute le nouveau point simulé à l'ensemble des points connus (incluant points conditionnants et points déjà simulés). On visite le point suivant et ainsi de suite jusqu'à ce qu'on ait visité tous les points à simuler. Cette procédure construit une réalisation de la variable. Pour atteindre l'objectif fixé dans le cas présent à 200 réalisations, une à chaque noud de la grille, d'autres chemins aléatoires sont choisis et la procédure est répétée.

Seules les catégories d'artefacts présentant un variogramme structuré ont fait l'objet d'une simulation conditionnelle Gaussienne. A partir des 200 réalisations obtenues, des cartes de probabilité de dépassement de seuil destinées à faciliter la lecture de la répartition des artefacts ont été produites. 


\section{Tests paramétriques sur les moyennes}

Des tests paramétriques sur les moyennes d'abondance, de teneur et d'altitude ont été effectués pour caractériser certaines zones par rapport à leur voisinage immédiat. Ces tests ont été réalisés à l'aide du logiciel XLSTAT ${ }^{\oplus}$. Etant donnée la taille des échantillons comparés (effectif de chaque échantillon $>30$ individus), le test $Z$ est utilisé pour réaliser les comparaisons des moyennes. Ce test $Z$ ne nécessite pas l'égalité des variances des populations dont proviennent les échantillons.

Les hypothèses testées sont identiques à celles du test $\mathrm{t}$ de Student pour des échantillons indépendants. Les échantillons 1 et 2 sont prélevés respectivement dans deux populations d'espérances $\mu 1$ et $\mu 2$. Le test bilatéral correspond au test de la différence entre $\mu 1$ et $\mu 2$, et les hypothèses nulle $(\mathrm{H} 0)$ et alternative $(\mathrm{H} 1)$ sont les suivantes :

$-\mathrm{H} 0: \mu 1-\mu 2=0$

$-\mathrm{H} 1: \mu 1-\mu 2 \neq 0$.

\section{RÉSUlTATS ET INTERPRÉTATIONS}

\section{Répartition des artefacts au sol d'après les cartes d'estimation spatiale}

Conformément à la méthode d'estimation spatiale adoptée, un variogramme expérimental a été calculé pour chaque catégorie d'artefacts collectée.

Le calcul des variogrammes expérimentaux pour chaque catégorie a révélé que seules la céramique des I $^{\text {er }}$-III ${ }^{e}$ siècles, les TCA indéterminées et les tuiles à crochet étaient structurées spatialement. L'examen des variogrammes directionnels des autres catégories d'artefacts révèle la présence d'une anisotropie : l'ajustement d'un modèle est alors effectué sur le variogramme expérimental réalisé dans la direction de cette anisotropie.

Dans l'ensemble, l'effet de pépite est plutôt faible ce qui suggère que la maille de mesure est suffisamment petite et que la distribution des artefacts est relativement bien structurée dans l'espace. En revanche, la variabilité de l'effet de pépite d'une catégorie à l'autre (de 14 à $78 \%$ ) signifie que l'on n'explique pas la même proportion de la distribution spatiale des artefacts selon les catégories considérées.

Pour réaliser les cartes, le nombre d'artefacts collecté par cellule de ramassage est attribué à un point qui se situe au centre de la cellule échantillonnée. Ainsi, les valeurs estimées lues en chacun des points de la carte correspondent aux quantités d'artefacts présents dans une cellule d'échantillonnage ( $10 \mathrm{~m} \times 3 \mathrm{~m})$.
Les cartes d'estimation spatiale obtenues à l'issue de l'analyse géostatistique offrent une représentation de la répartition des artefacts très proche de la répartition réelle au sol (fig. 5). Elles mettent en évidence des zones de forte abondance révélant non seulement la présence de structures archéologiques enfouies - notamment antiques - dans certains cas mais également des phénomènes de surface que nous cherchons à caractériser.

\section{Cartes de probabilité de dépassement de seuil}

Les seuils, définis comme la quantité moyenne d'artefacts estimée dans l'emprise de chaque cellule, ont été déterminés à partir de la moyenne et de la médiane d'abondance de chaque catégorie d'artefacts. Les valeurs supérieures au seuil estimé sont interprétées comme les indices potentiels de la présence de structures archéologiques en sous-sol. Ainsi, le seuil a été fixé à 5 artefacts pour la céramique $\mathrm{I}^{\mathrm{er}}-\mathrm{III}^{\mathrm{e}}$ siècles, 2 artefacts pour la tuile à crochet et 7 artefacts pour la TCA indéterminée. Les autres catégories d'artefacts étant nettement moins représentées, le seuil pour la simulation a été fixé à 1 artefact par cellule.

Les résultats les plus concluants ont été obtenus pour le clos, parcelle pour laquelle on dispose de données complémentaires issues des prospections géophysique et géochimique. La simulation conditionnelle a permis en particulier de circonscrire, pour la céramique $\mathrm{I}^{\mathrm{er}}-\mathrm{III}^{\mathrm{e}}$ siècles, six zones de fortes probabilités de dépasser un seuil susceptibles de signaler la présence de structures enfouies (fig. 6).

Ces zones de fortes probabilités correspondent, dans l'ensemble, aux anomalies détectées en prospection géophysique interprétées comme des structures artisanales liées à l'officine de potier antique (fours et/ou fosses-dépotoirs).

\section{Redistribution de la matière sur les versants}

\section{Combinaison des cartes d'abondance}

Si les zones de forte abondance d'artefacts mises en évidence dans le clos révèlent la présence de structures archéologiques sous-jacentes, le reste du terrain prospecté offre une image de la répartition des artefacts plus contrastée dont l'interprétation est moins liée à la présence de vestiges enfouis qu'aux phénomènes de redistribution post-dépôt.

La combinaison des cartes des artefacts les plus abondants - céramique $\mathrm{I}^{\mathrm{er}}-\mathrm{III}^{\mathrm{e}}$ siècle, TCA indéterminée, tuiles à crochet - permet de circonscrire précisément les zones présentant systématiquement moins de mobilier, quelle que soit la catégorie d'artefacts considérée (fig. 7). Hormis les effets de bordures dus à l'échantillonnage, deux zones de faible abondance se dessinent clairement : l'une dans la parcelle de la Butte, l'autre au centre de la parcelle de la Robinerie. 

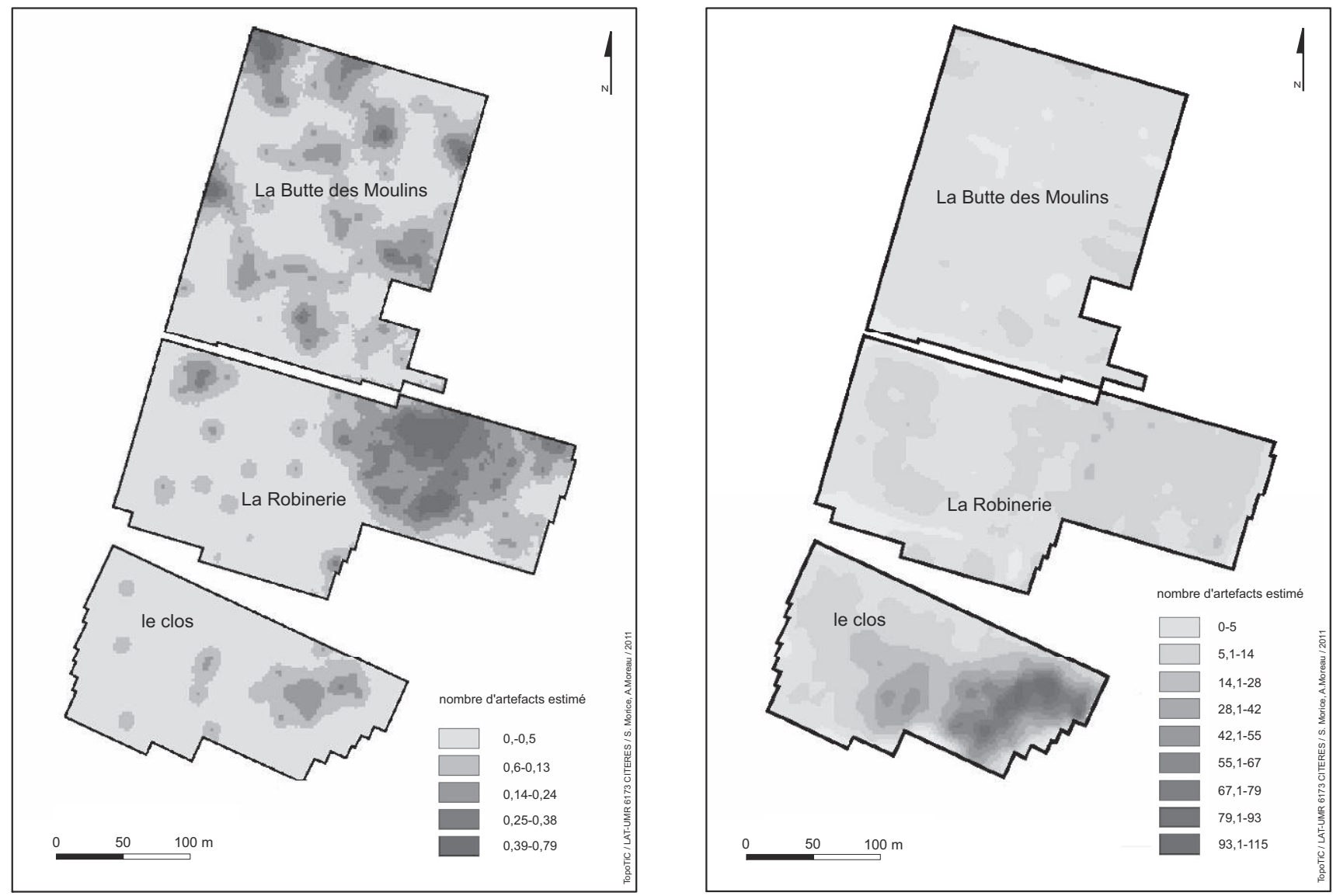

Figure 5 : Cartes de distribution spatiale des artefacts obtenue par krigeage (gauche : céramique protohistorique, droite : céramique $\mathrm{I}^{\mathrm{er}}$-III ${ }^{\mathrm{e}}$ siècle). Figure 5 : Spatial distribution of artifacts drown by kriging (left: protohistoric pottery; right: pottery of the $1^{\text {st }}$ - $3^{\text {rd }}$ century)

Moins de 7 artefacts ont été estimés par cellule dans ces deux zones de faible abondance.

\section{Tests paramétriques $Z$ :}

\section{caractérisation de la parcelle de la Robinerie}

Les données géochimiques et topographiques disponibles pour la parcelle de la Robinerie (Zadora-Rio et al., 2008) ont permis de mieux caractériser la zone de vide relatif d'artefacts observée au centre de cette parcelle.

Afin de déterminer si la différence de moyenne d'artefacts par cellule entre la zone de vide relatif de la Robinerie et les zones voisines est réellement significative, le test paramétrique Z, effectué à partir du nombre d'artefacts calculé au sein de chaque cellule d'échantillonnage, a été réalisé pour deux échantillons. Les résultats sont présentés dans le tableau 1.

On constate que, pour toutes les catégories à l'exception de la céramique protohistorique et de la céramique $\mathrm{XII}^{\mathrm{e}}$ $\mathrm{XIII}^{\mathrm{e}}$ siècles, il y a significativement moins d'artefacts dans la « zone centrale » de la parcelle de la Robinerie qu'en périphérie. Le même test paramétrique $Z$, effectué à partir des valeurs des teneurs géochimiques estimées, révèle que les teneurs moyennes des éléments chimiques sont significativement plus faibles dans la "zone centrale" qu'alentour. Seul le calcium présente une tendance inverse avec un seuil plus élevé dans la zone centrale. Enfin, le test $\mathrm{Z}$ appliqué aux données de convexité indique que la "zone centrale " se distingue du reste de la parcelle par son aspect convexe.

Les différences de valeurs constatées entre le calcium - aux teneurs particulièrement importantes dans la zone centrale et les autres éléments traces ainsi que les valeurs de susceptibilité magnétique (Zadora-Rio et al., 2008) beaucoup moins élevées dans la zone centrale qu'alentour, peuvent être attribuées à une évolution des sols plus ou moins poussée. Le substrat étant crayeux, le processus de pédogenèse dominant est la décarbonatation. Or, une décarbonatation peu ou moins avancée induit une plus forte abondance de calcium total au détriment des autres éléments et des oxydes qui 


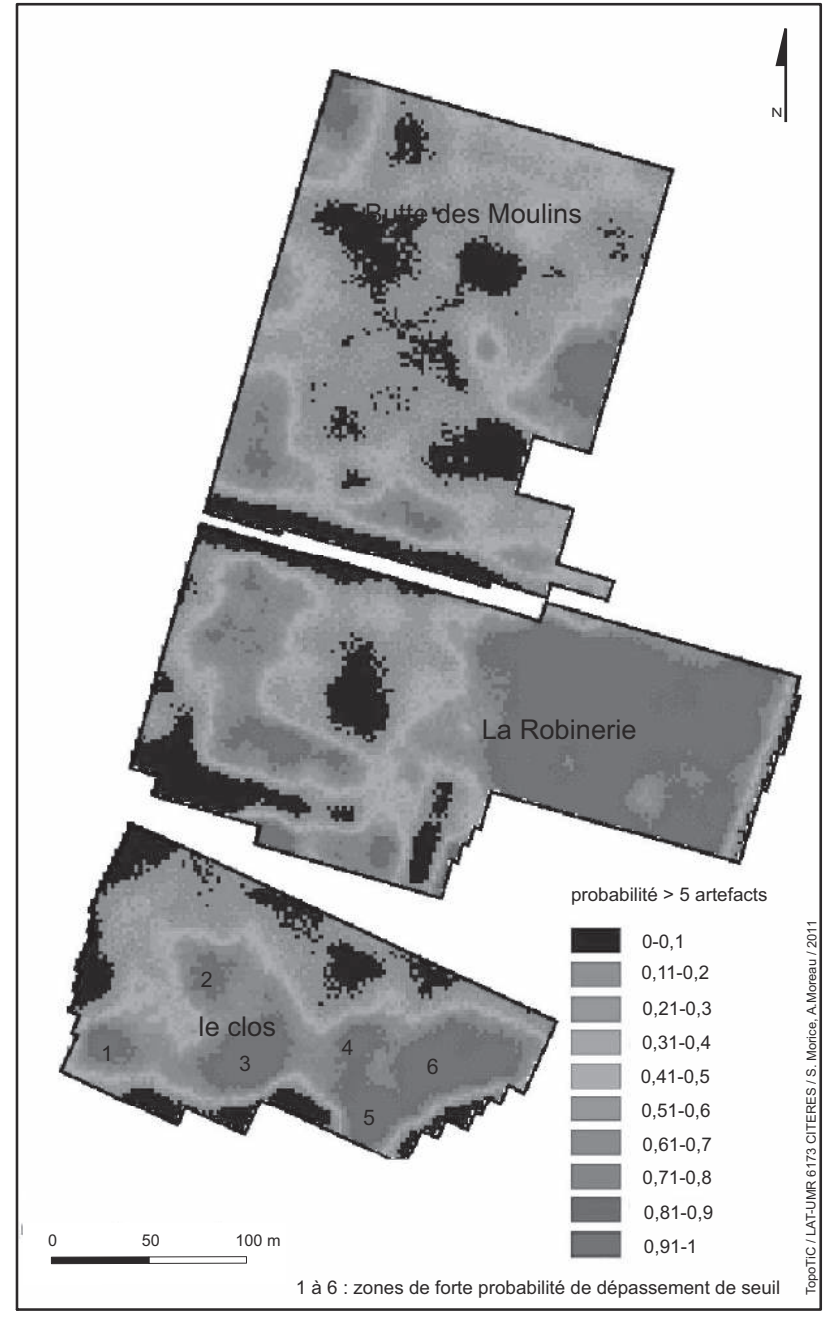

Figure 6: (Voir planche couleur) Carte de forte probabilité de dépassement de seuil, céramique ${ }^{\mathrm{er}}{ }^{-} \mathrm{III}^{\mathrm{e}}$ siècle, obtenue par simulation séquentielle gaussienne.

Figure 6: (See colour plate) Places where the density threshold is likely to be exceeded, pottery of the $1^{\text {st }}-3^{\text {rd }}$ century drown by sequential gaussian simulation.

se concentrent progressivement lors de la décarbonatation. Ainsi, la « zone centrale » présente toutes les caractéristiques d'une zone aux sols moins évolués, peu épais et riches en carbonates ce qui est à l'origine de la " tache claire » interprétée initialement $d$ 'après les photographies aériennes verticales comme un bâtiment gallo-romain potentiel.

Le rajeunissement des sols couplé à l'absence relative d'artefacts dans cette même zone tendent alors à témoigner d'une redistribution de la matière et des artefacts après leur dépôt. Dans un milieu comme celui qui est considéré ici, les deux agents érosifs susceptibles d'être à l'origine de la redistribution de la matière et du rajeunissement des sols de la

\begin{tabular}{|c|c|c|}
\hline Catégorie & $\begin{array}{l}\text { Moyenne zone } \\
\text { centrale }\end{array}$ & Moyenne alentour \\
\hline Céramique protohistorique & $0,02 \mathbf{a}$ & $0,07 \mathbf{a}$ \\
\hline Céramique I ${ }^{\mathrm{er}}-\mathrm{III}^{\mathrm{e}}$ siècle & $2,35 \mathbf{a}$ & $7,68 \mathbf{b}$ \\
\hline Céramique $\mathrm{IV}^{\mathrm{e}}-\mathrm{VII}^{\mathrm{e}}$ siècle & $0,01 \mathbf{a}$ & $0,08 \mathbf{b}$ \\
\hline Céramique $\mathrm{VIII}^{\mathrm{e}}-\mathrm{XII}^{\mathrm{e}}$ siècle & $0,01 \mathbf{a}$ & $0,17 \mathbf{b}$ \\
\hline Céramique $\mathrm{XII}^{\mathrm{e}}$-XIII ${ }^{\mathrm{e}}$ siècle & $0,17 \mathbf{a}$ & $0,23 \mathbf{a}$ \\
\hline Céramique $\mathrm{XIV}^{\mathrm{e}}-\mathrm{XV}^{\mathrm{e}}$ siècle & $0,09 \mathbf{a}$ & $0,17 \mathbf{b}$ \\
\hline Céramique $\mathrm{XVI}^{\mathrm{e}}$-XVII ${ }^{\mathrm{e}}$ siècle & $0,05 \mathbf{a}$ & $0,17 \mathbf{b}$ \\
\hline Céramique XviI ${ }^{\mathrm{e}}$ siècle et plus & $0,21 \mathbf{a}$ & $0,43 \mathbf{b}$ \\
\hline Ardoise & $0,21 \mathbf{a}$ & $0,59 \mathbf{b}$ \\
\hline TCA indéterminée & $2,31 \mathbf{a}$ & $11,33 \mathbf{b}$ \\
\hline Tuile à crochet & $0,42 \mathbf{a}$ & $5,36 \mathbf{b}$ \\
\hline Calcium & $19,37 \mathbf{b}$ & $6,38 \mathbf{a}$ \\
\hline Potassium & $0,04 \mathbf{a}$ & $0,89 \mathbf{b}$ \\
\hline Aluminium & $0,50 \mathbf{a}$ & $2,16 \mathbf{b}$ \\
\hline Fer & $0,58 \mathbf{a}$ & $1,28 \mathbf{b}$ \\
\hline Manganèse & $0,02 \mathbf{a}$ & $0,05 \mathbf{b}$ \\
\hline Phosphore & $0,02 \mathbf{a}$ & $0,05 \mathbf{b}$ \\
\hline Silicium & $18,23 \mathbf{a}$ & $30,10 \mathbf{b}$ \\
\hline Titane & $0,12 \mathbf{a}$ & $0,18 \mathbf{b}$ \\
\hline convexité verticale & $-0,032 \mathbf{a}$ & $0,165 \mathbf{b}$ \\
\hline convexité horizontale & $0,020 \mathbf{a}$ & $-0,042 \mathbf{a}$ \\
\hline
\end{tabular}

Tableau 1 : Test $Z$ de différence des moyennes des catégories d'artefacts, des teneurs géochimiques et des mesures de convexité (les lettres en gras indiquent une différence significative ou non au seuil $\alpha=0,05$ ).

Table 1: $Z$ test of difference between the averages of artifacts categories, geochemical properties and convexities.

zone centrale sont l'érosion hydrique ou le labour. La remobilisation des artefacts par érosion hydrique semble toutefois peu probable : la plupart des artefacts ont une taille pluricentrimétrique qui rend difficile leur déplacement par l'érosion hydrique diffuse. Par ailleurs, rien n’indique la présence de ravines ou de rigoles. La remobilisation de la matière est donc plus vraisemblablement le fait des labours répétés que de l'érosion hydrique. La forte convexité verticale de la zone considérée vient conforter cette dernière hypothèse : des travaux ont montré que les opérations de labour des contextes topographiques complexes ont tendance à provoquer le départ de matière depuis les zones convexes, le dépôt se faisant en contexte concave (Lobb, Kachanoski, 1999).

ArcheoSciences, revue d'archéométrie, 35, 2011, p. 103-116 


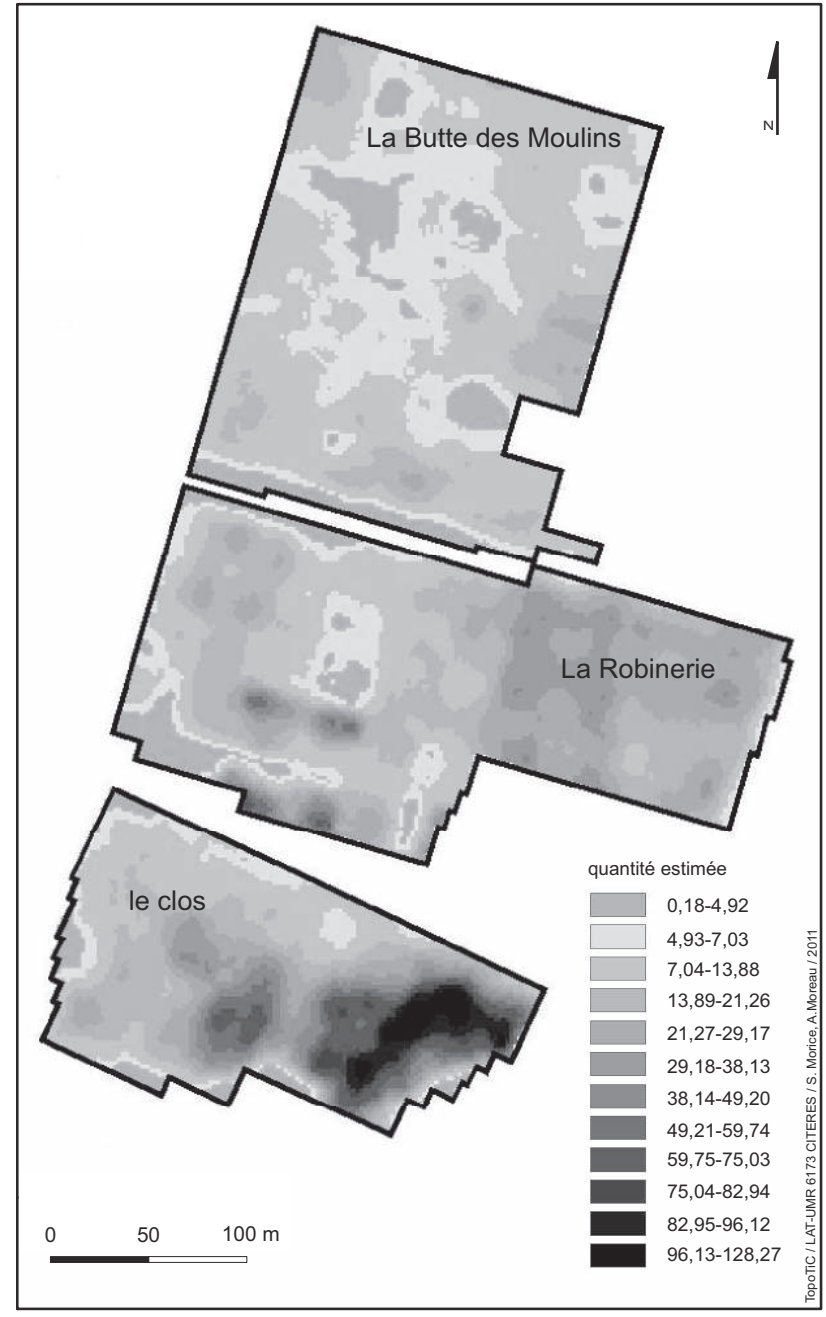

Figure 7 : (Voir planche couleur) Combinaison des cartes d'abondance de la céramique $\mathrm{I}^{\mathrm{er}}$-III ${ }^{\mathrm{e}}$ siècle, de la TCA indéterminée et de la tuile à crochet.

Figure 7: (See colour plate) Combination of the maps showing the amounts of pottery of the $1^{\text {st }}-3^{\text {rd }}$ century, building materials and modern tiles.

\section{MODES DE REPRÉSENTATION DES DONNÉES ET CONFRONTATION DES IMAGES DE SURFACE}

Afin de juger de la pertinence de l'unité d'enregistrement et de représentation des artefacts (la cellule), les données ont été cartographiées en Nombre de Restes par cellule puis en Nombre de Restes par carré de $30 \mathrm{~m}$ x $30 \mathrm{~m}$. Les cartes de distribution des artefacts par carré ont été ensuite confrontées aux cartes de répartition en Nombre de Restes par cellule et aux cartes d'estimation réalisées à l'issu du traitement géostatistique. Les résultats sont riches d'enseignement : les zones de vide et d'accumulation d'artefacts sont particulièrement mises en relief sur les représentations en Nombre de Restes par carré, qu'il s'agisse des zones où la présence de vestiges est supposée (le clos) ou des zones de remobilisation de la matière par les labours ("vide central » et accumulation orientale de la Robinerie). Aucune information d'importance ne semble omise par rapport aux cartes de répartition à l'échelle de la cellule et aux cartes d'estimation spatiale obtenues par traitement géostatistique.

Cette confrontation permet de discuter non pas uniquement de la taille de la maille de ramassage mais aussi du mode de représentation spatiale des données : on passe de la cellule au carré mais la précision du ramassage est celle de la cellule. Cette expérience montre que la représentation des données à une échelle plus grossière que l'échelle de collecte (le carré de $30 \times 30 \mathrm{~m}$ ) n'est pas moins instructive qu'une représentation à l'échelle de la maille de collecte : la représentation par carré offre finalement une image de surface très proche de l'image obtenue après traitement géostatistique, plus facile à lire et à interpréter d'un point de vue archéologique qu'une représentation par cellule.

\section{Conclusion}

Ce constat nous amène à formuler trois conclusions préliminaires relatives aux méthodologies de collecte et d'exploitation des données de surface :

- on a généralement tendance à considérer que plus l'échelle d'enregistrement des données est précise, meilleur est le contrôle de l'information; dans le cas présent, on constate que la cellule de $3 \times 10 \mathrm{~m}$ n'est pas nécessairement une unité de représentation des données pertinente dans le sens où elle ne révèle rien de plus que ce qui nous est donné à voir à l'échelle des carrés de 30 x $30 \mathrm{~m}$; ce constat est finalement peu surprenant : dans la mesure où l'image de surface est brouillée par les processus post-dépôt, il est vain de vouloir lire les informations de surface avec la même précision que l'on déchiffre les données du sous-sol;

- ainsi, si les données de prospection ne font pas l'objet d'un traitement géostatistique, il n'est pas forcément utile d'enregistrer voire de collecter les données à une échelle aussi précise;

- par ailleurs, si l'on reste au stade de la simple distribution des données de surface, la géostatistique ne montre rien de plus que ce que l'on observe avec la répartition en Nombre de Restes; certes, l'application des méthodes d'estimation spatiale contribue à restituer fidèlement l'image réelle de surface en minimisant la variance d'estimation lors de l'interpolation ce qui, en effet, facilite la lecture des don- 
Figure 8 : (Voir planche couleur) Répartition des Terres Cuites Architecturales en NR par cellule (a), NR par carré (b) et après traitement géostatistique (c).

Figure 8: (See colour plate) Distribution of building materials (number of pieces) in each "cellule" (a), each square(b) and after geostatistical analysis.
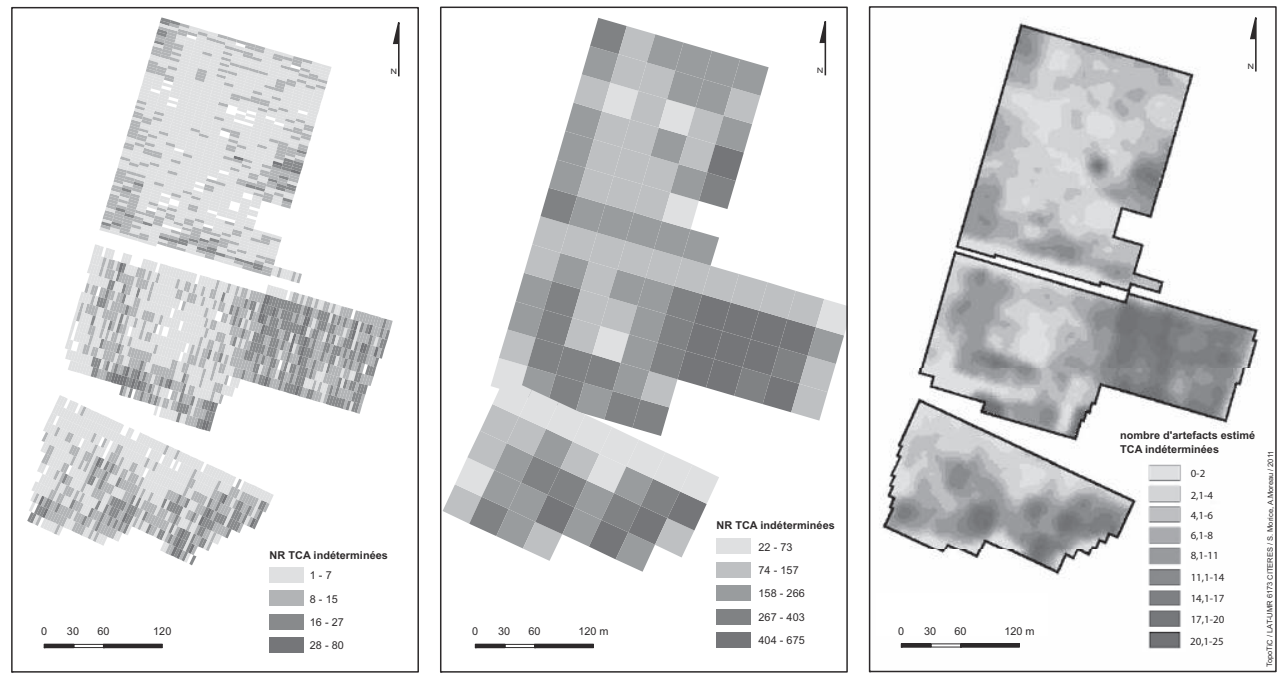

nées sans toutefois, apporter d'information supplémentaire par rapport à la répartition en Nombre de Restes par cellule.

Cependant, l'emploi de la géostatistique ne se limite pas à l'élaboration des cartes d'estimation et l'exploitation des données de surface par l'étude géostatistique offre des perspectives particulièrement intéressantes :

- les cartes de probabilités de dépassement de seuils ont ainsi permis de circonscrire très précisément les zones potentiellement riches en vestiges archéologiques; dans le cas de la parcelle du clos, la distribution des artefacts des Ir-III $^{e}$ siècles telle qu'elle apparaît sur la carte de probabilité de dépassement de seuil confirme largement les résultats de la prospection magnétique (Zadora-Rio et al. 2008) : on suppose alors que la superposition des anomalies géophysiques et des concentrations à forte probabilité dessine l'emplacement précis des structures archéologiques;

- la combinaison des cartes d'abondance présente l'avantage de prendre en compte plusieurs catégories de mobilier simultanément, mettant ainsi en évidence les zones récurrentes de vide documentaire (absence d'artefacts) interprétées à partir des données topographiques et géochimiques comme des zones plus érodées par les labours.

En définitive, qu'il s'agisse de délimiter plus précisément l'emplacement des structures archéologiques enfouies ou de mettre en évidence les manifestations de la redistribution de matière liées aux labours, l'utilisation de la géostatistique, justifiée par la précision de la maille de collecte, s'est révélé pertinente. S'il ne semble guère possible d'aller plus avant dans la détection des vestiges archéologiques, l'étude de la redistribution de la matière mériterait d'être développée davantage par l'étude fine du poids des artefacts afin de quantifier les mouvements de matière sur les versants. Les résultats obtenus pourraient être intégrés à des modèles de redistribution de matière par le labour et contribueraient ainsi à mieux évaluer l'érosion associée à ce travail de la terre.

\section{Bibliographie}

AlCAYDÉ, G. 1977. Notice et carte géologique au 1/50000 de Sainte-Maure-de-Touraine (XVIII-24), éd. BRGM, Orléans.

Bourassé, J.-J., 1854. Tombeaux de l'époque mérovingienne découverts en Touraine, Mémoire de la Société Archéologique de Touraine, VI, p. 237-244.

Castrignano, A. et Buttafuoco, G., 2004. Geostatistical Stochastic Simulation of Soil Water Content in a Forested Area of South Italy. Biosystems Engineering, 87, p. 257-266.

Chevalier, 1871. Communications, séance du 22 Nov.1871 et séance du 25 Nov.1872, Bulletin de la Société Archéologique de Touraine, II, 1871, p. 72-p.246.

Cinnirella, S., Buttafuoco, G. et Pirrone, N., 2005. Stochastic analysis to assess the spatial distribution of groundwater nitrate concentrations in the Po catchment (Italy), Environmental Pollution, 133, p. 569-580.

Couderc, J.-M., 1982. Nouveaux sites antiques en Touraine et nouvelles données sur des sites connus, Bulletin de la Société Archéologique de Touraine, 40, p. 77-88.

DuвoIs, J., 1985. Prospections 1983 et 1984 en Touraine, Bulletin de la Société Archéologique de Touraine, XLI, p. 97-114.

Ferdière, A., 1999. Mougon, dans Bellet, M.-E., Cribellier, C., Ferdière, A., Krausz, S. (dir). Agglomérations antiques en 
Région Centre, $17^{e}$ supplément à la Revue Archéologique du Centre de la France, vol.1, 1999, p. 139-144.

Ferdière, A. et Zadora-Rio, E. (dir.), 1986. La prospection archéologique, Paysage et peuplement. Actes de la table ronde des 14 et 15 mai 1982. Document d'Archéologie Française, Éditions de la Maison des Sciences de l'Homme, Paris.

FoARD, G., 1978. Systematic fieldwalking and the investigation of Saxon settlement in Northamptonshire, World Archaeology, 9, $\mathrm{n}^{\circ} 3$, p. 357-374.

Lobb, D. A. et KaChanoski, R. G., 1999. Modelling tillage erosion in the topographically complex landscapes of southwestern Ontario, Canada, Soil and Tillage Research, 51, p. 261-277.
Moreau, A., 2008. Du tesson au système territorial : une approche multiscalaire de l'occupation du sol dans la vallée de la Vienne autour de L'Île-Bouchard (Indre-et-Loire). Thèse de doctorat d'histoire, Université de Tours, France.

SCHOFIELD, A.-J., 1991. Interpreting artefacts scatters, contributions to ploughzone archaeology. Oxbow Monograph 4, Oxbow Books, Oxford.

Zadora-Rio, E., Ferdière, A., Buko, A., Tabbagh, A. et Verhaeghe, F. (dir.), 2008. La dynamique des paysages et des sociétés pré-industrielles : approche comparative et intégrée des marqueurs du changement contenus dans le sol, Rapport de fin de programme ACI Terrain, Technique, Théories. 